ARTICLE

DOI: $10.1038 / \mathrm{s} 41467-017-00543-2$

OPEN

\title{
A 3D magnetic tissue stretcher for remote mechanical control of embryonic stem cell differentiation
}

Vicard Du ${ }^{1}$ Nathalie Luciani ${ }^{1}$, Sophie Richard ${ }^{1}$, Gaëtan Mary${ }^{1}$, Cyprien Gay ${ }^{1}$, François Mazuel ${ }^{1}$, Myriam Reffay ${ }^{1}$, Philippe Menasché ${ }^{2}$, Onnik Agbulut ${ }^{3} \&$ Claire Wilhelm ${ }^{1}$

The ability to create a 3D tissue structure from individual cells and then to stimulate it at will is a major goal for both the biophysics and regenerative medicine communities. Here we show an integrated set of magnetic techniques that meet this challenge using embryonic stem cells (ESCs). We assessed the impact of magnetic nanoparticles internalization on ESCs viability, proliferation, pluripotency and differentiation profiles. We developed magnetic attractors capable of aggregating the cells remotely into a 3D embryoid body. This magnetic approach to embryoid body formation has no discernible impact on ESC differentiation pathways, as compared to the hanging drop method. It is also the base of the final magnetic device, composed of opposing magnetic attractors in order to form embryoid bodies in situ, then stretch them, and mechanically stimulate them at will. These stretched and cyclic purely mechanical stimulations were sufficient to drive ESCs differentiation towards the mesodermal cardiac pathway.

\footnotetext{
${ }^{1}$ Laboratoire Matière et Systèmes Complexes (MSC), UMR 7057, CNRS and Université Paris Diderot, 75205 Paris Cedex 13, France. ${ }^{2}$ Department of Cardiovascular Surgery, Hôpital Européen Georges Pompidou; Paris Cardiovascular Research Center, INSERM U970, Université Paris Descartes, Paris 75015 France. ${ }^{3}$ Sorbonne Universités, UPMC Univ Paris 06, Institut de Biologie Paris-Seine (IBPS), UMR CNRS 8256, Biological Adaptation and Ageing, 75005 Paris, France. Correspondence and requests for materials should be addressed to C.W. (email: claire.wilhelm@univ-paris-diderot.fr)
} 
$\mathrm{R}$ esearch in regenerative medicine has advanced rapidly over the past decade thanks to the development of multiple tools (e.g., 3D printing and 3D culture, controlled forces and microenvironments, cell differentiation and reprogramming $)^{1-4}$. Stem cells and their unique potential for differentiation lie at the heart of this emerging field.

In particular, a growing number of studies have evidenced that mechanical factors can influence stem cell differentiation ${ }^{5}$. This idea of a physical guidance of differentiation emerged from studies using adult mesenchymal stem cells, and was then tested on pluripotent/embryonic stem cells. Most techniques applied on two-dimensional (2D) cell cultures, focusing in particular on the role of microenvironmental mechanical cues such as substrate rigidity $^{6-11}$, flow-induced shear stress ${ }^{12-14}$, strains imposed on cell monolayers by the stretching of deformable supporting membranes ${ }^{15-17}$, or local forces applied on beads attached to the cell surface ${ }^{18,19}$.

Multicellular three-dimensional (3D) approaches have also received an increasing interest for studying stem cell behavior beyond the classical 2D culture conditions. First, scaffold-based constructions not only allow to stimulate mechanically the seeded stem cells ${ }^{20}, 21$, but also provide precise $3 \mathrm{D}$ control of extracellular matrix cues 22,23 . Second, scaffold-free magnetic or printing technologies make it possible to control spatial patterning of aggregates ${ }^{24}$ or to create multilayer structures ${ }^{25}$.

One current challenge is now to provide other methodologies to assemble and organize stem cells (only) into a 3D tissue structure that can be stimulated at will, in order to explore the physical differentiation approaches in 3D purely cellular tissues.

To create a $3 \mathrm{D}$ cell assembly, one needs to enable remote spatial organization of component cells. Magnetic cellular forces acting at a distance are appealing candidates for this application, provided the individual cells are first magnetized by the internalization of magnetic nanoparticles. Magnetic nanoparticles in regenerative medicine are mostly used either for noninvasive in vivo tracking of stem cells by magnetic resonance imaging ${ }^{26-29}$, or for magnetic cell targeting to sites of tissue damage $\mathrm{d}^{21,30-32}$. The idea of using magnetic cell manipulation for tissue engineering is more recent, and the first works featured bioprinting and cell sheet engineering, by magnetically creating or manipulating spheroids ${ }^{33-35}$ or organizing layers of several cell types $^{36,37}$, respectively. To use magnetic forces not only to form tissues, but also to remotely stimulate them, is still to be unraveled.

Incorporating nanoparticles to magnetize and stimulate cells raises several issues. The first is the impact of nanoparticle internalization on the cell phenotype, and particularly differentiation capacity. Previous studies ${ }^{31,38}$ of mesenchymal stem

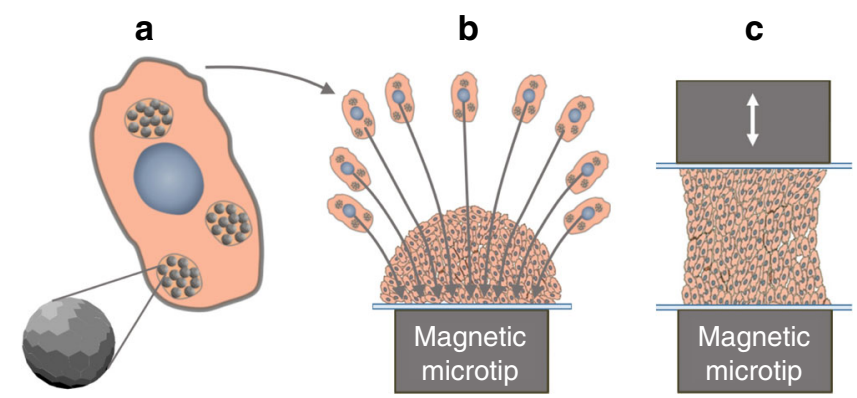

Fig. 1 Schematic illustrating the different steps involved in the magnetic stretcher. a Nanoparticles incorporation in ESCs, b EBs formation from magnetized ESCs driven by a magnetic microtip, and $\mathbf{c}$ EBs magnetic stimulation in situ, in the 3D geometry, and without the need for a supporting matrix cells have shown that magnetic nanoparticles generally do not inhibit their differentiation, except for chondrogenesis in some cases $^{39}$, in particular at high iron doses ${ }^{40}$. Besides, magnetic nanoparticles can also be beneficial to mesenchymal stem cells differentiation, e.g., for myocardial repair ${ }^{41,42}$. Only few studies have investigated the impact of magnetic nanoparticles on embryonic stem cells (ESCs). One reported that cardiomyogenesis was unaffected ${ }^{43}$, another that the self-renewal ability or surface phenotypic markers expressed after forced differentiation into hematopoietic cells were unchanged ${ }^{44}$. To the best of our knowledge, the impact of magnetic nanoparticles on the whole ESC differentiation profile, with no biochemical triggers, is still unknown.

ESC differentiation is initiated within an embryoid body (EB), generally created with the hanging drop method. A second important question is thus whether 3D magnetic printing of ESCs could be equivalent to this method and what would be its impact on the differentiation profile after cell maturation. The ultimate and most challenging question is whether magnetic forces alone could drive stem cells differentiation within a magnetically formed 3D model tissue.

Here we address all three issues by using magnetized ESCs to create an EB and remote magnetic forces to stimulate it (Fig. 1). We first carefully analyzed iron oxide nanoparticle internalization by ESCs, and its impact on their viability, pluripotency and differentiation. Second a magnetic attraction method was developed to create EBs, and its impact on the ESC differentiation profile was evaluated. We then designed an all-in-one magnetic stretcher capable of both creating and stimulating the EB and evidenced the impact of purely mechanical stimulation on $\mathrm{EB}$ differentiation.

\section{Results}

ESCs magnetic labeling, viability and pluripotency. The first step was to incorporate magnetic nanoparticles within ESCs. Magnetophoretic measurements ${ }^{40}$ of the iron load per cell (Fig. 2a) yielded uptake curves (in terms of pg of iron per cell) that saturated as a function of both the extracellular iron concentration $[\mathrm{Fe}]$ and the incubation time. Thus, by setting the incubation time to $30 \mathrm{~min}$, a saturation value of $3.3 \pm 0.5 \mathrm{pg}$ was obtained with $[\mathrm{Fe}]=2 \mathrm{mM}$. When the concentration was set at $[\mathrm{Fe}]=2 \mathrm{mM}$, a saturation value of $6.6 \pm 0.5 \mathrm{pg}$ was obtained after 2-4 h. Nanoparticle uptake can be directly imaged with Perls' Prussian blue staining (Fig. $2 \mathrm{~b}$ and Supplementary Fig. 1 for other views). Observation of cell colonies showed that the stronger the labeling, the more intense the blue color, up to saturation, correlating well with the magnetophoretic measurements. Finally, transmission electron microscopy of labeled cells showed that the nanoparticles were all internalized and localized within lysosomes; no nanoparticles were observed outside the cells (see Fig. 2c and Supplementary Fig. 2 for other views).

Because ESCs are particularly sensitive to perturbations, the possibility that nanoparticles might impact their viability and proliferation had to be first considered. To test the viability, ESCs metabolic activity was measured after magnetic labeling in different conditions (iron masses between 1.8 and $6.6 \mathrm{pg}$ per cell). It is important to note that the cells must be incubated in RPMI medium with $5 \mathrm{mM}$ citrate to prevent nanoparticle aggregation before cell internalization. It is therefore also necessary to quantify the impact of citrate itself on cell viability. Figure $2 \mathrm{~d}$ shows cell viability for all the conditions tested, as compared to the control (unlabeled cells in culture medium). The short labeling period of $30 \mathrm{~min}$ had no impact at 0.5 or $2 \mathrm{mM}$ extracellular iron, but a slight effect (about -10\%) was noted at $5 \mathrm{mM}$ iron. Upon increasing the labeling period, viability fell at all 
iron concentrations. However, the viability of the cells incubated with citrate alone was modified in the same way as during magnetic labeling. Citrate alone thus impacted cell viability, whereas the nanoparticles had only a slight impact on viability at high iron concentrations. Cell viability was also examined on the long-term, over 9 days after labeling for the $30 \mathrm{~min}$ incubation condition ( 3 pg per cell), and no impact was observed on the cells' viability and ability to replicate compared to control cells (Supplementary Fig. 3).

The next step was to analyze the impact of magnetic labeling on ESC pluripotency. The first indicator of ESC pluripotency is their morphology; undifferentiated cells form round colonies. As shown in Supplementary Fig. 4, ESC colonies remained well-rounded after labeling under different conditions. Figure $2 \mathrm{e}$

a
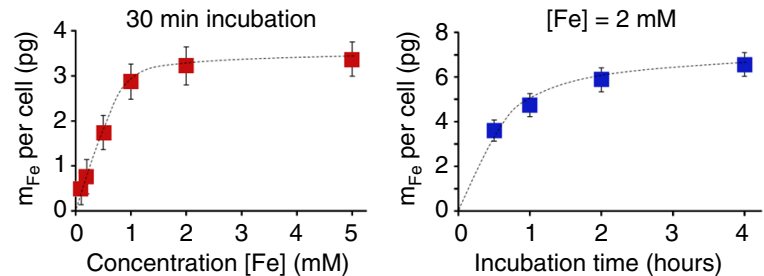

b

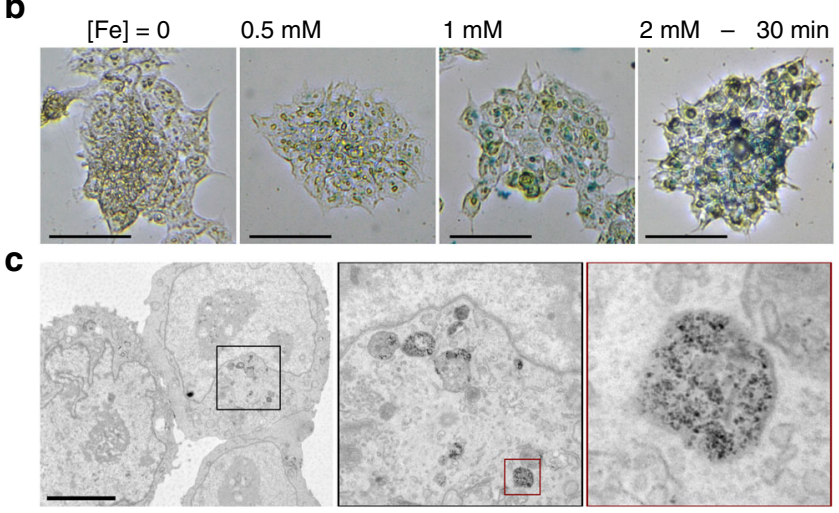

d

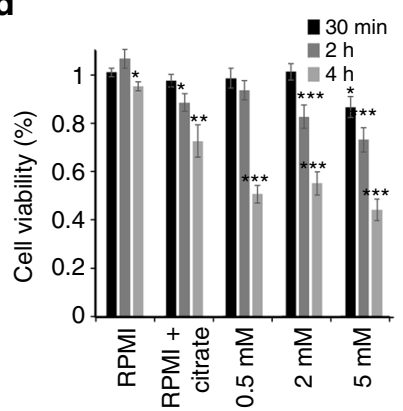

e

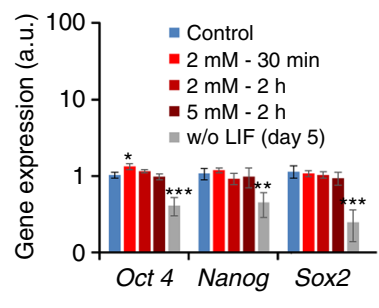

f

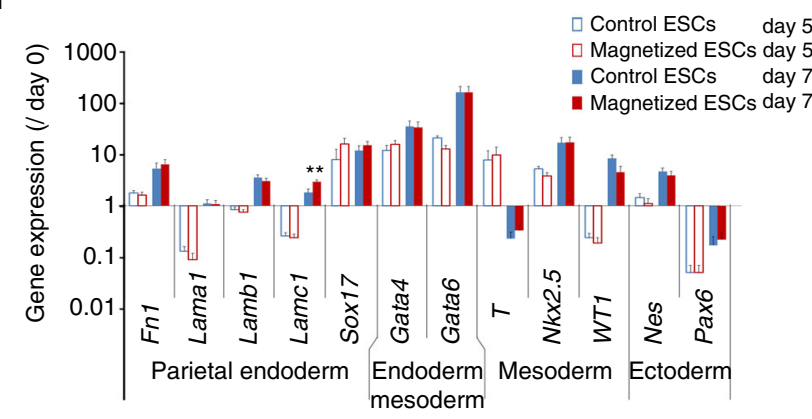

shows that the expression level of the key pluripotency genes Oct 4, Nanog and Sox 2 were unaffected by the internalization of the nanoparticles, whatever the labeling condition, as compared to the control (unlabeled cells). By contrast, the positive control (culture without the leukemia inhibitor factor LIF, essential to maintain pluripotency) suffered a significant decrease of these genes at day 5 . In view of these different results, the labeling condition of $30 \mathrm{~min}$ at $[\mathrm{Fe}]=2 \mathrm{mM}$ was chosen for subsequent experiments, as it yielded an iron mass of 3 pg per cell without affecting viability, proliferation, morphology and pluripotency. Hereafter, the resulting ESCs are referred to as magnetized ESCs.

Impact of magnetic labeling on differentiation profile. The next mandatory step was to retrieve the differentiation profile of EBs formed with magnetized ESCs, and compare it with the one of control EBs (unlabeled ESCs). We chose the standard hanging drop EB formation method to initiate differentiation. This method begins with the seeding of 1000 ESCs within a $30 \mu$ drop, which spontaneously assembles into an EB. Supplementary Fig. 5 shows images of EB formation with unlabeled control and magnetized ESCs. No difference in EB morphology was observed. mRNA from control EBs and magnetized EBs were then collected and analyzed on days 5 and 7. The expression levels (shown in Fig. 2f) of Fn1, Lama1, Lamb1, Lamc1, Sox17 (endoderm markers); T, Nkx2.5, Wt1 (mesoderm markers), Gata4, Gata6 (meso-endoderm markers); and Nes and Pax 6 (ectoderm markers) were measured by real-time PCR. Importantly, the obtained results showed no differences between control and magnetized EBs, demonstrating that the temporal expression pattern of the different genes was very similar.

Magnetic formation of EBs. Having established the optimal conditions for ESC magnetic labeling, the next challenge was to

Fig. 2 Optimization of embryonic stem cell (ESC) magnetic labeling. a Magnetic labeling of ESCs at different extracellular iron concentrations (for a fixed incubation time of $30 \mathrm{~min}$ ) and during different incubation periods (for a fixed iron concentration of $[\mathrm{Fe}]=2 \mathrm{mM}$ ). b Perls' Prussian blue staining of ESCs after labeling with different concentrations of extracellular iron (between $0.5 \mathrm{mM}$ and $2 \mathrm{mM}$ ), and a fixed incubation time of $30 \mathrm{~min}$. Scale bar: $250 \mu \mathrm{m}$. c Transmission electron micrograph of ESC after labeling for $30 \mathrm{~min}$ at $[\mathrm{Fe}]=2 \mathrm{mM}$ (successive zooms of framed areas). Scale bar: $5 \mu \mathrm{m}$. Nanoparticles are all located inside the lysosomes. d Cell viability testing using Alamar Blue detection of cell metabolic activity. Cell viability was calculated relative to the control (unlabeled cells in complete medium) and was measured $2 \mathrm{~h}$ after different incubation periods (in RPMI) with different extracellular iron concentrations and incubation times. e Expression of pluripotency genes Oct4, Nanog and Sox2 measured by real-time PCR. The gene expression level was calculated with respect to RPLPO mRNA and expressed as compared to control (unlabeled cells, cultured in complete medium with $L I F,=1 \pm S E M$ ). A positive control was added in which the LIF has been removed during 5 days before analysis (culture in complete medium without LIF). One can note that only one condition led to a significant upregulation (Oct4-incubation at $2 \mathrm{mM}$ for $30 \mathrm{~min}$ ). However the gene was upregulated $<1.5$-fold (1.3-fold exactly). Besides, higher doses ( $2 \mathrm{~h}$ incubation at 2 and $5 \mathrm{mM}$ ) provide the same Oct4 expression as the control. $\mathbf{f}$ Expression of several genes characteristic of the different embryonic layers in hanging drop EB formation conditions with 1000 unlabeled (control, blue bars) or labeled cells (magnetic, red bars), 5 days (open bars) and 7 days (solid bars) after initiation of differentiation. All values were calculated with respect to RPLPO MRNA and normalized by the expression value of the same gene measured at day 0 . Two-sample $t$-test was used to compare the control group with the magnetic group, for same gene and same day; ${ }^{\star} p<0.05$; ${ }^{\star \star} p<0.01$; ${ }^{\star \star \star} p<0.001$. All error bars represent the SEM 
create EBs magnetically, as an alternate method to hanging drop or others. The idea was simple: nanoparticles contained in cells confer a cellular magnetic moment $\mathrm{M}_{\text {cell }}$ (measured by magnetophoresis), which allows the cells to be attracted by a magnetic force $\mathrm{F}_{\mathrm{m}}=\mathrm{M}_{\text {cell }}$ gradB. In order to use this force to confine cells within a spheroid, gradB must be at submillimeter scales. To achieve this, we developed submillimetric $(750 \mu \mathrm{m}$ diameter $)$ metal tips to channel the magnetic lines of an external field (about $0.2 \mathrm{~T}$ ), creating a strong field gradient localized in space. Figure $3 \mathrm{a}$ shows ESC attraction by one magnetic microtip, perfectly matching the gradient map (Fig. $3 \mathrm{~b}$ ). The field gradient is $500 \mathrm{~T} \mathrm{~m}^{-1}$ at $1 \mathrm{~mm}$ from the surface of the microtip $\left(1000 \mathrm{~T} \mathrm{~m}^{-1}\right.$ at $0.4 \mathrm{~mm})$, equivalent to a force of about $100 \mathrm{pN}(200 \mathrm{pN}$, respectively) on an ESC containing 3 pg of iron.

Networks of these magnetic spikes were used to assemble several EBs in the same dish (Supplementary Fig. 6 explains in detail the fabrication of these magnetic devices). The microtips were spaced a few millimeters apart in order to preserve the localization of the field gradient created by each microtip. Magnetic formation of EBs was then straightforward (Supplementary Fig. 7): on day 0, the cells were labeled, detached and placed in a Petri dish (after non-adhesive treatment with PLL-PEG), itself placed over the magnetic microtip array.

a

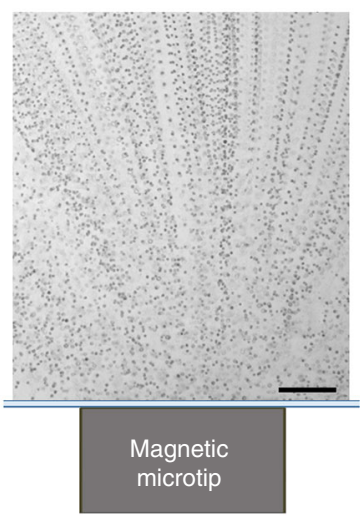

b

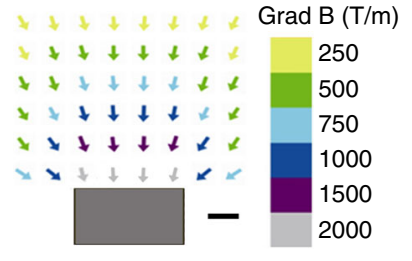

C

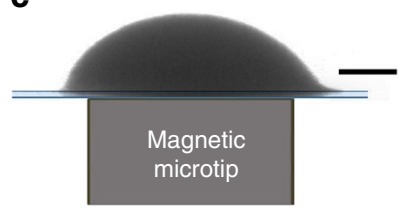

d

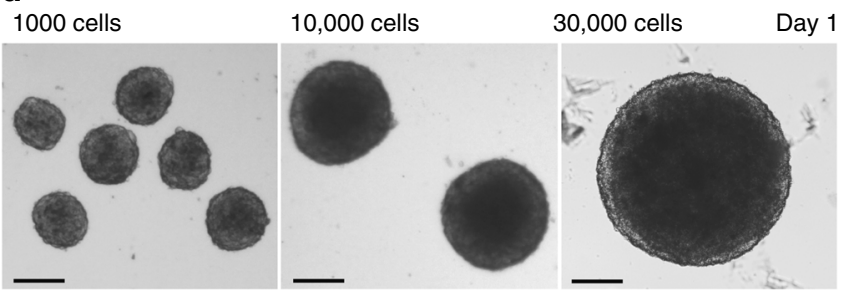

e Magnet
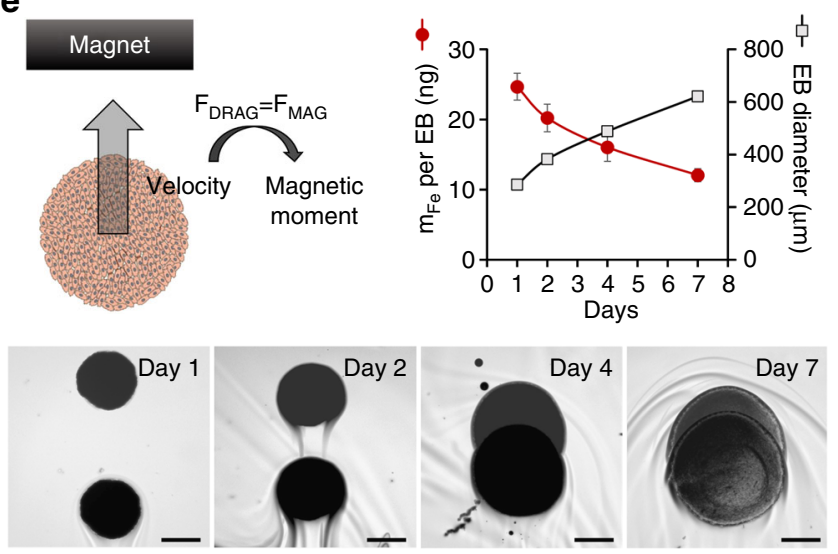

We tested 1000, 10,000 and 30,000 cells per microtip (and thus per EB). Figure $3 c$ shows EB formed from 30,000 cells, positioned over a microtip, right after deposition. Figure $3 \mathrm{~d}$ show the spheroids thus obtained, one day after cell deposition (day 1).

Long-term intracellular fate of the nanoparticles in the EBs. One essential question remains that of the fate of the nanoparticles once internalized within ESCs. Or alternatively, will the EB stay magnetic over long-term culture conditions? To address this issue, we monitored EBs' magnetism (initially 10,000 cells) at different times after EB formation, by magnetophoresis (Fig. 3e). Briefly, it consists in tracking the EB magnetic mobility when submitted to a homogeneous magnetic field gradient created by a permanent magnet. The magnetic velocity can then be directly converted into the EB magnetic moment, or alternatively the amount of nanoparticles (expressed in mass of iron) contained within the EB. At day 1 after formation, each EB contains on average $25 \mathrm{ng}$ of iron, consistent with the initial iron load per single ESC of about $3 \mathrm{pg}$. This amount progressively decreases during EB growth, reaching about half its initial value at day 7. This is due to the lysosomal degradation of the nanoparticles, as recently evidenced in MSC spheroids ${ }^{45}, 46$. While the degradation is beneficial for long-term ability of magnetically-labeled tissue to get rid of the initial nanoparticles, the fact that at day 7, EBs still retain half their magnetization is also beneficial for multiple magnetic stimulations before tissue maturation.

Magnetic EB formation versus hanging drop. This system of magnetic formation allows tight control of EB size, contrary to the hanging drop method, which yields EBs of more variable size and, in some cases, no EBs at all. Figure 4a shows the percent of EB successfully formed, the EB average diameter and ellipticity, for magnetic EB formation or hanging drop, starting from 1000 or 10,000 ESCs. Magnetic EB formation appears particularly advantageous when starting from 10,000 cells, where the success rate of formation increases from 73 to $91 \%$ when using magnetic formation instead of the hanging drop approach, and EB ellipticity decreases from 0.17 to 0.04 . The size control is also

Fig. 3 Magnetic formation of embryoid bodies. a ESC attraction by a magnetic microtip ( $750 \mu \mathrm{m}$ in diameter). To visualize cell movement, the microtip was introduced into a chamber containing suspended cells under a microscope, and cell movements were video-monitored with a $\times 10$ objective. Here 100 movie images were superimposed (0.1 s time intervals) in order to directly observe the trajectories of the cells migrating towards the magnetic microtip. At $1 \mathrm{~mm}$ from the microtip, the cells migrate at an average velocity of $300 \mu \mathrm{m} / \mathrm{s}$, which corresponds to an iron mass of $3 \mathrm{pg}$ cell in a magnetic field gradient of $300 \mathrm{mT} / \mathrm{mm}$. b The field gradient was mapped around the microtip by studying the migration of monodisperse magnetic beads with a calibrated diameter of $4.6 \mu \mathrm{m}$ (Dynal). At $1 \mathrm{~mm}$ from the microtip, it was $300 \mathrm{mT} / \mathrm{mm}$. Scale bar: $200 \mu \mathrm{m}$. c Final image of the aggregate obtained $1 \mathrm{~min}$ after seeding 30000 ESCs over the magnetic microtip. Scale bar: $200 \mu \mathrm{m}$. d Microscopic images of embryoid bodies (EBs) on day 1, obtained by seeding 1000, 10,000 and 30,000 cells per microtip. Scale bar: $200 \mu \mathrm{m}$. e Monitoring of EBs magnetism over 7 days after nanoparticles cellular incorporation, and EB formation (day 0 ). It consists of tracking the EB magnetic migration towards a magnet, and measuring the corresponding velocity, which translates into the $E B$ magnetic moment (proportional to the mass of iron per EB) by balancing the viscous drag and the magnetic force. Typical migrations are shown for the different times (days 1, 2, 4 and 7), corresponding to the superimposition of two images at $3 \mathrm{~s}$ interval. Scale bar: $200 \mu \mathrm{m}$. The mass of iron (circles) and the EBs diameters (squares), averaged over eight different EBs, were then plotted as a function of time. Error bars represent the SEM 
a

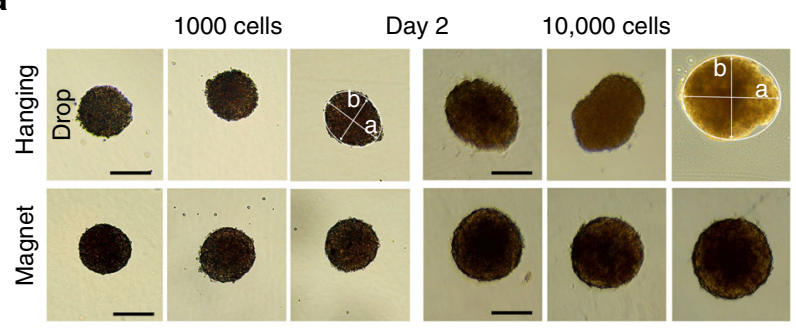

b

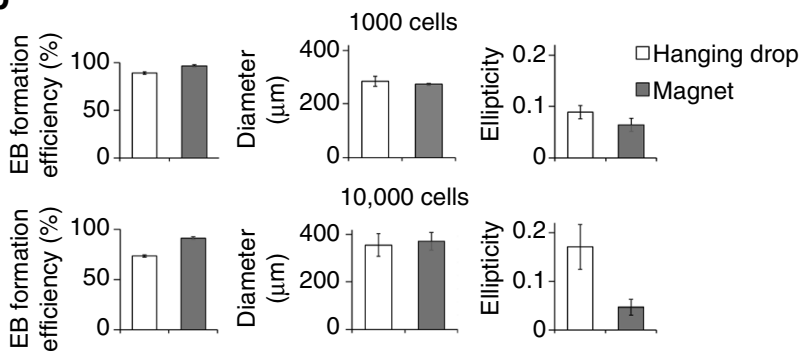

C
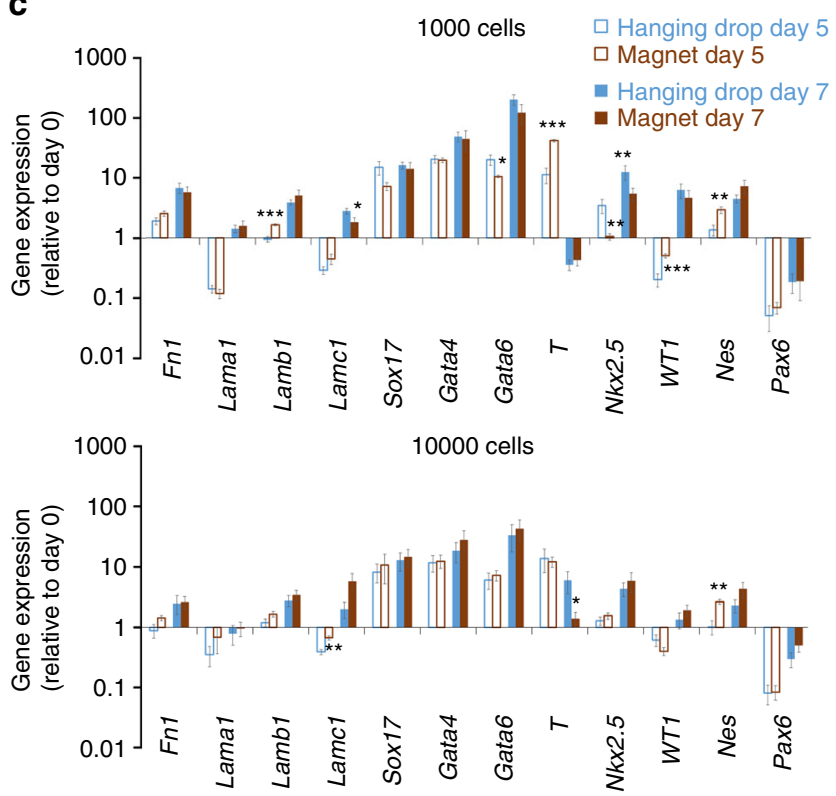

Fig. 4 Comparison between magnetic EB formation and hanging drop method. a Typical images of EBs observed at day 2 after seeding (of 1000 or 10,000 ESCs), either in hanging drop or over a magnetic attractor. The short $a x i s b$ and the long-axis $a$ of the equivalent ellipse were determined by image analysis (Image J). Scale bar: $200 \mu \mathrm{m}$. b Quantification over 50 EBs: Efficiency is calculated as the number of EBs actually formed over the number of hanging drops deposited or of magnetic attractors present below the dish; the diameter (expressed in $\mu \mathrm{m}$ ) is the effective diameter computed from the EBs areas; and the ellipticity is defined as 1-b/a. c Expression of a panel of genes characteristic of the different embryonic cell layers in EB obtained with hanging drop or magnetic aggregation with either 1000 or 10,000 cells. All gene expressions were normalized using the reference gene RPLPO mRNA, and calculated relatively to the expression of the same gene obtained at day 0 , prior to EB formation. The values varied very little from one condition to another. Even statistically significant differences (over- or under-expression) were small: with 1000 cells on day 5, Lamb1 expression increased by a factor of 1.7 and $T$ expression by 3.7, while $N k \times 2.5$ fell by a factor of 3.2, Wt1 rose by 2.5 and Nes rose by 2.1. On day 7, Lamc1 expression fell 1.5-fold and Nkx2.5 fell 2.3-fold. With 10,000 cells, Lamc1 increased 1.7-fold and Nes 2.6-fold on day 5, while T fell 4.2-fold on day 7. All error bars represent the SEM increased as demonstrated by a thinner distribution of EBs sectional areas in case of magnetic formation (see Supplementary Fig. 8). Finally, magnetic EB formation is almost instantaneous, and the transfer step (drops to dishes on day 2) necessary for the hanging drop method is avoided.

We then studied ESC differentiation under these magnetic conditions of $\mathrm{EB}$ formation. As previously, differentiation is initiated at day 0 of magnetic EBs formation by LIF removal from culture medium. Figure $4 \mathrm{~b}$ compares the magnetic formation technique (magnet condition) to the hanging drop method, for 1000 and 10,000 cells deposited per EB, on day 5 and day 7 of maturation. The expression level of most of the genes involved in the three embryonic layers of the EBs formed magnetically was unchanged compared to control EBs formed with the hanging drop. Significant differences were observed in only 10 of the 48 conditions, and significant differences observed for one gene are never spread to all conditions (from day 5 to day 7, or from 1000 cells to 10,000 cells).

Magnetic stretcher for in situ EB formation and stimulation. ESCs thus retain a similar differentiation profile as obtained in control condition, not only after incorporating magnetic nanoparticles, but also after magnetic formation of EBs. The concept of the magnetic stretcher then becomes relevant: the EB should be formed in the culture medium by the same magnetic microtip as described above, then another magnetic microtip would be approached to trap and deform (stretch/stimulate) it (Fig. 5a), and only this mechanical stimulation step would be responsible for any change in differentiation capacity.

The magnetic stretcher (Fig. 5a and Supplementary Fig. 9) combines fixed and mobile microtips for stretching and stimulation, in a perfectly sterile setup. With dimensions $(20 \times 40 \times 20 \mathrm{~cm})$, it fits easily inside an incubator, to maintain the EBs temperature at $37^{\circ} \mathrm{C}$, with $5 \% \mathrm{CO}_{2}$. It was also designed to be placed directly on a microscope stage, thermalized at $37^{\circ} \mathrm{C}$, without compromising sterility. The magnetic microtips are inserted in machined structures closed by glass slides $100 \mu \mathrm{m}$ thick to prevent contact between the metal and the culture medium, while allowing them to be very close to the cells. Removable cylindrical magnets are used to magnetize the microtips. The device allows three EBs to be formed and stimulated at the same time, and medium reservoirs (communicating with the rest of the setup) are placed between the microtips to ensure nutrient distribution to the EBs. A micrometric motor (Thorlabs, controller interfaced with Labview) ensures the normal motion of one of the structures carrying the magnetic devices (microtips and magnets), while two micrometric displacements ensure alignment along the other two axes.

The EBs are initially formed from 10,000 cells on the fixed magnetic structure (Fig. 5b). These EBs correspond to the magnet condition, similar to the one shown in Fig. 3c, which were found to be identical in terms of differentiation profile to EBs formed with hanging drop and 10,000 ESCs. As the second microtip is approached, $1 \mathrm{~h}$ after EB formation, the EB is deformed between the two tips (Fig. 5b). This corresponds to the stretched condition, for which, during the following 3 days, each EB is maintained in its stretched configuration. Finally the cyclic condition corresponds to an additional cyclic stimulation (at a frequency of $1 \mathrm{~Hz}$ and an amplitude of 10\%) for two $2 \mathrm{~h}$ periods each day of the following 3 days (day 1, day 2 and day 3; Fig. 5c).

In this setup, the magnitude of the magnetic (intracellular) force applied to single ESCs within the magnetic EB needs first to be quantified. At $400 \mu \mathrm{m}$ from the magnetic tip/attractor and for ESCs loaded with $3 \mathrm{pg}$ of iron (or equivalently a magnetic moment of $2 \times 10^{-13} \mathrm{~A} \mathrm{~m}^{2}$ ), the magnetic gradient of about 
$1000 \mathrm{~T} / \mathrm{m}$ provides a force of approximately $200 \mathrm{pN}$ per single ESC. More precisely, the magnetic force is exerted on the nanoparticles clustered within lysosomes. Each lysosome is thus submitted to a force of $1 \mathrm{pN}$ since it contains a maximum of $10^{4}$ nanoparticles and bears a magnetic moment in the range of $10^{-15} \mathrm{~A} \mathrm{~m}^{2}$. Lysosomes, which are embedded in the viscoelastic cytoplasm, are observed not to agglomerate onto each other or drift significantly within the cell, see Supplementary Fig. 10 which shows that the intracellular pattern of the magnetic lysosomes is the same with or without magnet application.

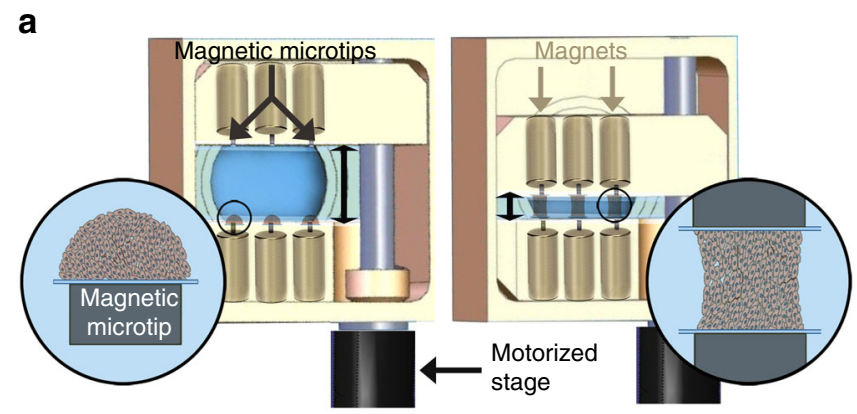

b

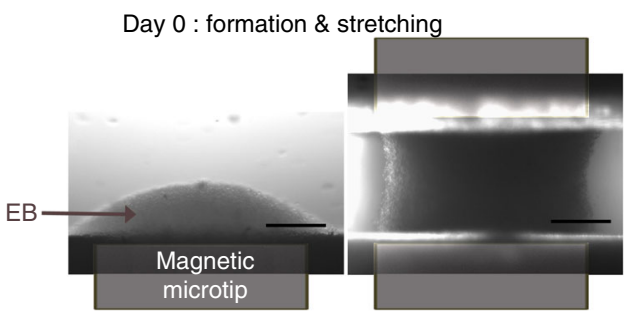

C

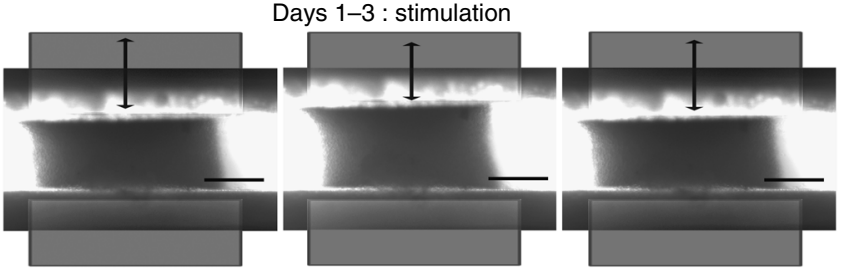

d

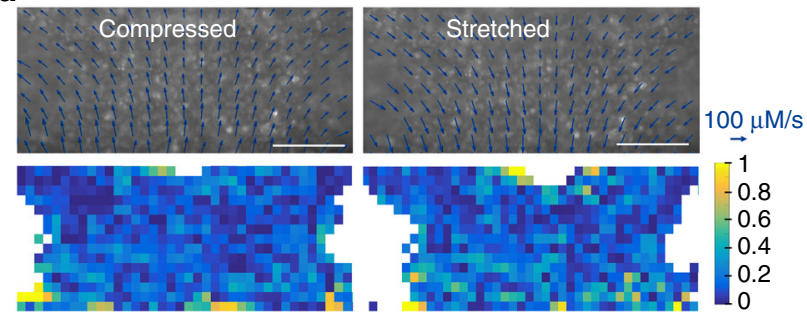

e

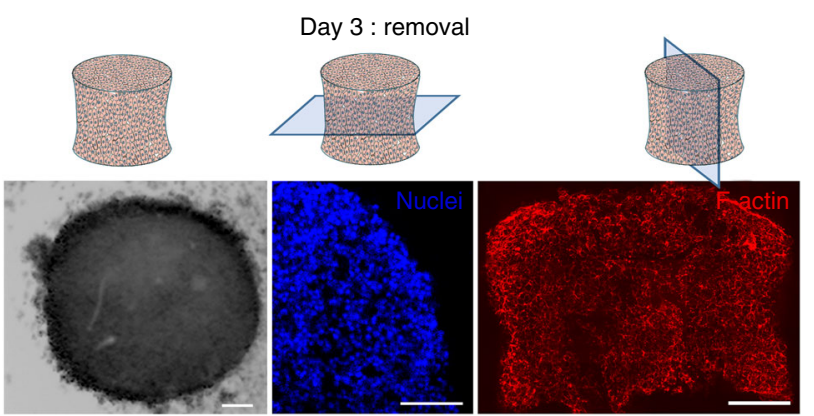

To document the stimulation at single-cell levels, cell movements were monitored over several stretching cycles using a membrane cell marker (Pkh26). PIV analysis provided the velocity field of the cells submitted to stretching and compression due to magnet displacements (Fig. 5d). No shear zones are noticeable on this figure. Moreover cells inside the EBs are submitted to a uniform strain over the whole aggregate. Indeed the divergence of the velocity field which is representative for the strain rate r $^{48}$ is homogenous. The average effective strain rate is $0.32 \pm 0.08 \mathrm{~s}^{-1}$ for the stretching step and $0.32 \pm 0.06 \mathrm{~s}^{-1}$ for the compression step. Thus all cells experience essentially the same deformation rate.

Finally, in all conditions, at the end of day 3, the spheroids could be released (Fig. 5e) by removing the permanent magnets (thus canceling the magnetic force), and transferred into dishes to mature until day 5 before mRNA collection and analysis. The fact that each EB was easily released as soon as the microtips were demagnetized shows that the stretching is due to remote forces, exerted at the heart of the tissue structure, with no direct contact. Besides, as demonstrated in Fig. 5e on cryosections parallel and perpendicular to the tissue axis, the EB maintains its engineered shape.

Figure 6a shows the expression levels of genes characteristic of the different embryonic layers, under the three conditions (magnet, stretched and cyclic), and at day 5. All levels are expressed relatively to the same genes expression at day 0 , right before EB formation and LIF removal. The expression levels of the stretched and cyclic conditions must thus be compared with the ones of the magnet condition, which are almost identical to the control hanging drop method. First, we can note an increase in the expression of $N k x 2.5$ involved in the cardiac mesoderm pathway for the stretched condition, enhanced for the cyclic condition. Concerning the other mesoderm gene $T$, because the decrease in the expression of this gene has already begun at day 5 (see Supplementary Fig. 11 for the timing of $T$ and $N k \times 2.5$ involvement in cardiac differentiation), upregulation is lesser, nevertheless increased for the stretched and cyclic conditions. Second, we also measured a significant increase of the 3 genes involved in the next stage towards cardiac differentiation, Sox17, Gata4 and Gata6 (Supplementary Fig. 11 also summarizes the role of these genes in cardiac differentiation), compared to the control magnet condition, and this increase was higher for the

Fig. 5 Magnetic stretcher: formation and stimulation of EBs. a Diagram of the magnetic stretcher device developed. Three EBs could be created on magnetic microtips (magnetized by permanent magnets), and then could be stretched/stimulated by approaching another 3 microtips (also magnetized by permanent magnets). The system is motorized to realize micrometer displacement of the second mobile magnetic microtips system. b Typical images of the first phases on day 0 : EB formation and stretching. Scale bar: $200 \mu \mathrm{m}$. c Typical images of the cyclic stimulation (here at days 1-3). Scale bar: $200 \mu \mathrm{m}$. d Fluorescence images of membrane-stained cells in compressed and stretched EBs (10\% imposed strain) are overlaid with velocity vectors extracted from PIV analysis (arrow bar scales for a speed of $100 \mu \mathrm{m} / \mathrm{s}$ ). Only one fourth of the vectors are represented for easy reading. Scale bar: $100 \mu \mathrm{m}$. The divergence of the velocity field (for stretching) or its opposite (for compression) representative for the strain rate is mapped in both cases. For compression and stretching steps the mean effective strain rate sensed by cells is calculated at $0.32 \pm 0.08$ and $0.32 \pm 0.06 \mathrm{~s}^{-1}$, respectively. e EB sampling on day 3 (here shown for a "cyclic" condition): Optical microscopy right after magnet removal and fluorescent imaging (DAPI staining, middle; F-actin staining, right) of $16-\mu \mathrm{m}$ cryosections in the perpendicular and parallel direction of the tissue axis. The nuclei image shows a homogeneous cell density in the center of the EB, while $\mathrm{F}$-actin is homogenous whatever the localization of the cell inside the stretched EB. Scale bar: $100 \mu \mathrm{m}$. All EBs were formed with 10,000 ESCs 
a

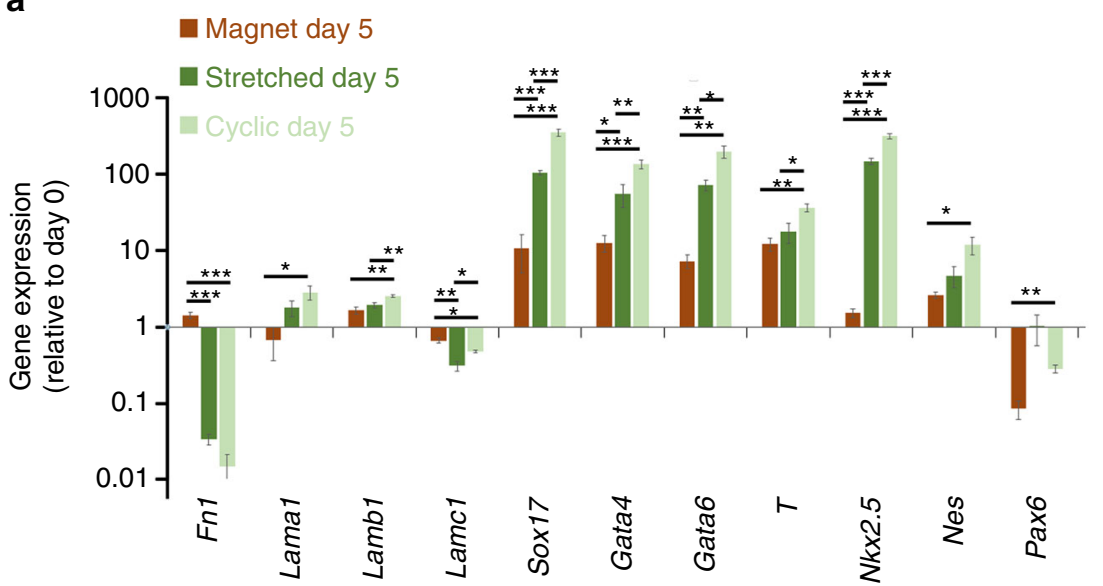

b
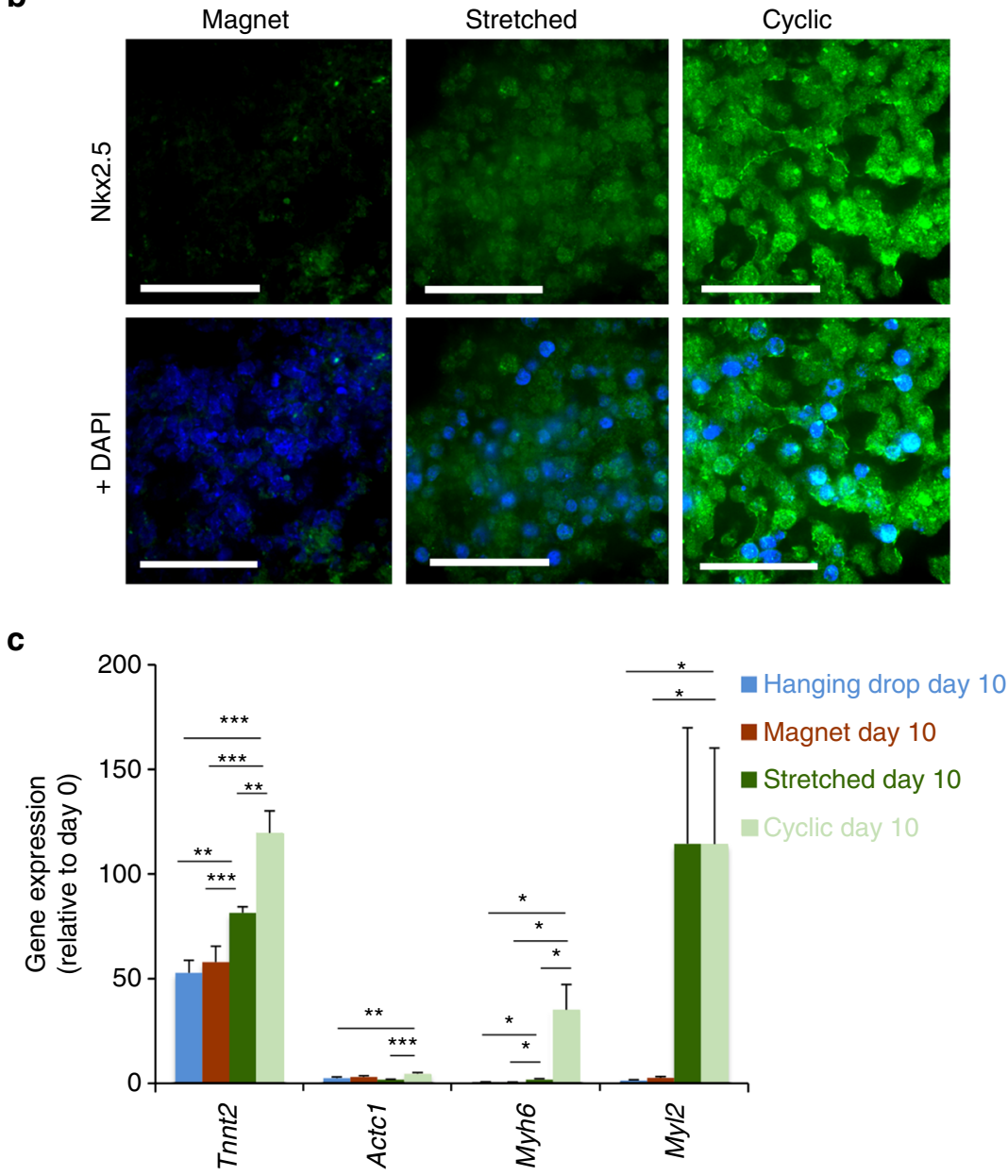

Fig. 6 EBs characterization for the magnet, stretched and cyclic conditions. a Expression of genes characteristic of the different embryonic cell layers in EB after 5 days maturation (day 5). All EBs were obtained from 10,000 magnetized ESCs. Magnet: EB created on a magnetic microtip; Stretching: EB formed on a magnetic microtip, then stretched between two microtips; Cyclic: as before, plus stimulation at $1 \mathrm{~Hz}$ twice a day for 3 days. Gene expression (normalized to RPLPO) is calculated relative to the same gene expression at day 0 before EB formation. $\mathbf{b}$ Immunostaining (in green) of Nkx2.5 for EBs in the three conditions, with DAPI staining overlaid on the right. Images are obtained at the center of each EB. Scale bar: $50 \mu \mathrm{m}$. c Gene expression at longer maturation times (day 10) for specific cardiac markers cardiac troponin T (Tnnt2), cardiac $\alpha$-actin (Actc1), $\alpha$ myosin heavy chain (Myh6) and myosin regulatory light chain 2 (Myl2). All EBs were obtained from 10,000 ESCs. For the hanging drop formation (blue), ESCs were not labeled with the magnetic nanoparticles. For the three other conditions, ESCs were magnetic ( $3 \mathrm{pg}$ of iron per cell): EB formation by magnet with no further stimulation (dark red), stretched stimulation (dark green) and cyclic stimulation (light green). mRNA levels are shown relative to control (day 0, defined as 1), and normalized to reference gene RPLPO. Error bars represent the SEM 
cyclic condition. Finally, and logically, the expressions of other genes involved in the endoderm or ectoderm pathways, were either lowly upregulated, with levels close to the ones for undifferentiated cells (Lama1, Lamb1, Lamc1, Nes and Pax6) or down-regulated (Lamc1 and Fn1).

Immunostaining of the Nkx2.5 protein confirmed these results (Fig. 6b). Its detection increased markedly in the stretched condition, and even more strongly after cyclic stimulation.

Finally, in order to detect if a commitment towards the cardiac lineage was really enhanced, we analyzed EB at longer maturation times (day 10, Fig. 6c), and quantified by qPCR the expression of transcripts encoding for specific cardiomyocyte markers. We tested troponin $\mathrm{T}$ (Tnnt2), involved in cardiomyocyte contraction, cardiac $\alpha$-actin (Actc1), the cardiac cytoskeletal marker, $\alpha$ myosin heavy chain (Myh6), involved in contraction and considered as a maturation marker, and myosin regulatory light chain 2 ( Myl2), involved in the regulation of myosin ATPase activity and known as a ventricular cardiomyocyte marker. The hanging drop and magnet conditions of EBs formation led to similar results for all genes and, overall, the efficiency of differentiation towards functional cardiomyocytes was increased upon application of stretched and cyclic stimulations. Compared to the magnet condition, Tnnt2, Myh-6 and Myl2 genes were overexpressed for the stretched condition, and this upregulation was higher following cyclic stimulation for Tnnt2 and Myh-6. The impact on cardiac $\alpha$-actin was less pronounced, with a significant upregulation only for the cyclic condition. This protein is, among others involved in the left ventricular compaction ${ }^{49}$, and probably expressed later.

\section{Discussion}

The main objective of this work was to provide a method for assembling embryonic stem cells into 3D embryonic bodies without the need for a scaffold and further stimulating mechanically this embryoid body in situ, with the overriding aim to determine whether embryonic stem cell differentiation could be enhanced in this $3 \mathrm{D}$ setting through mechanical stimulation. The engineering approach we propose is a magnetic one, in which magnetic forces are created intracellularly on internalized magnetic nanoparticles and used to manipulate single ESC within a $3 \mathrm{D}$ construct, to produce $3 \mathrm{D}$ embryoid bodies with an inherent capacity for further physical stimulation.

First, one must insist on the fact that, while ESCs are particularly vulnerable to external perturbations, yet, their differentiation profile after magnetic nanoparticle incorporation was remarkably preserved. This was mandatory for the next steps of EBs formation and stimulated differentiation. This result must also be put into perspective with the ongoing use of magnetic nanoparticles for regenerative medicine. Indeed, ahead of this application, the question of magnetic nanoparticles on stem cells differentiation must be addressed, and yet rarely was. Most works investigated impact on mesenchymal stem cells differentiation, with controversial results: some showed no effect on differentiation $^{31}$ while others showed an inhibition of chondrogenetic differentiation pathway ${ }^{50}$. One possible cause of this inhibition seems to be the cellular iron dose, with no impact at a low dose and inhibition at higher doses ${ }^{40}$. Here we provide a comprehensive analysis of the impact of magnetic nanoparticles on the whole differentiation profile of embryonic stem cells, and we evidence a striking preservation of this profile, boding well for their medical applications.

Studies of ESC differentiation require the creation of $3 \mathrm{D}$ multicellular aggregates (embryoid bodies). There are three main conventional approaches to $\mathrm{EB}$ formation in vitro ${ }^{51}$ : suspension culture with spontaneous aggregate formation; encapsulation in hydrogel; and the hanging drop method. The latter is the most widely used but it is time-consuming and requires multiple transfer steps. Control of EB size is a fundamental issue, as it plays an important role in differentiation, as demonstrated with alternative methods using microfabrication to control the size, cell number and shape of $\mathrm{EBs}^{52-55}$. Here we propose another alternative method for in situ EB formation on a magnetic attractor, with no impact on the differentiation profile when compared to the hanging drop technique. In addition, the equivalence between the two methods is robust, as demonstrated by the passage from 1000 cells to 10,000 cells per EB: with both the hanging drop method and the magnetic method, a fall in the expression of endoderm and mesoderm marker genes (especially the $T$ gene on day 5) was observed as EB size increased, in keeping with the few studies that have examined the influence of $\mathrm{EB}$ size on ESC differentiation ${ }^{52,54,56}$. The magnetic method of EB formation could thus be an interesting alternative to conventional techniques, especially as it avoids most of the manipulations associated with the hanging drop method.

In order to fully understand the formation and stimulation of the EB from a mechanical point of view, let us now examine the corresponding force balance in the magnetic stretcher apparatus, as depicted in Fig. 7.

During EB formation (Fig. 7a), the magnetic microtip subjects each cell to a magnetic force (blue arrow), pulling it against its neighbors, and thus contributing to squeezing all cells, but more strongly the ones closer to the microtip. The total resulting cellular magnetic force is then transmitted to the glass wall above the microtip, and is exactly balanced by the wall reaction force (green arrow). Straight after magnetic cell assembling, cohesion builds up through cell-cell junctions, and the whole assembly displays enough cohesion to be used as a standalone EB (Fig. 7b) and sustain stretching. Magnetic stretching is initiated by approaching a second magnetic microtip. The upper cell layers are then pulled against the upper wall (Fig. 7c). At each wall, the magnetic stretcher thus plays the role of a clamp acting on a proximal region of the sample held by the opposing magnetic force and the wall reaction force. At first, for a small distance between the microtips, the intermediate part of the $\mathrm{EB}$ is at rest while within each clamp, the wall reaction force exactly balances the corresponding total magnetic attraction force. Moving the clamps apart stretches the intermediate part of the EB and exerts pulling forces (black arrows) on the upper and lower (thin) proximal regions. Modulating the distance between the walls affects the degree of stretching of the large intermediate part (in a uniform manner as shown by the PIV measurements depicted in Fig. 5d) and the corresponding pulling forces and wall reaction forces. Meanwhile, the magnetic forces remain unchanged.

Let us now discuss whether the magnetic forces used to manipulate the magnetic EB can endanger the EB cohesion that results from cell-cell adhesion forces. Adhesive forces are generated by E-cadherin/E-cadherin bonds, measured at $73 \mathrm{pN}$ each, which amounts to about $900 \mathrm{nN}$ per mESC-mESC pair ${ }^{57}$. This is of the same order as the force required to separate two cells (several hundred $\mathrm{nN})^{58}$. One should compare this intensity to the highest tensile cell-cell forces within the aggregate. This happens to be within the intermediate region in the stretched configuration (Fig. 7c). The magnitude of the tensile force in this region is at most equal to that of the total magnetic force, around $1000 \mathrm{nN}$. This tensile force is distributed over all cells within a horizontal section of the aggregate (for instance the mid-height plane), corresponding to roughly 500 cells. It yields a typical maximum tensile force of $2 \mathrm{nN}$ per cell pair, safely below the mESC-mESC separation force.

It is also important to emphasize that, using only one magnetic microtip does not alter significantly the gene expression (Fig. 4b), 

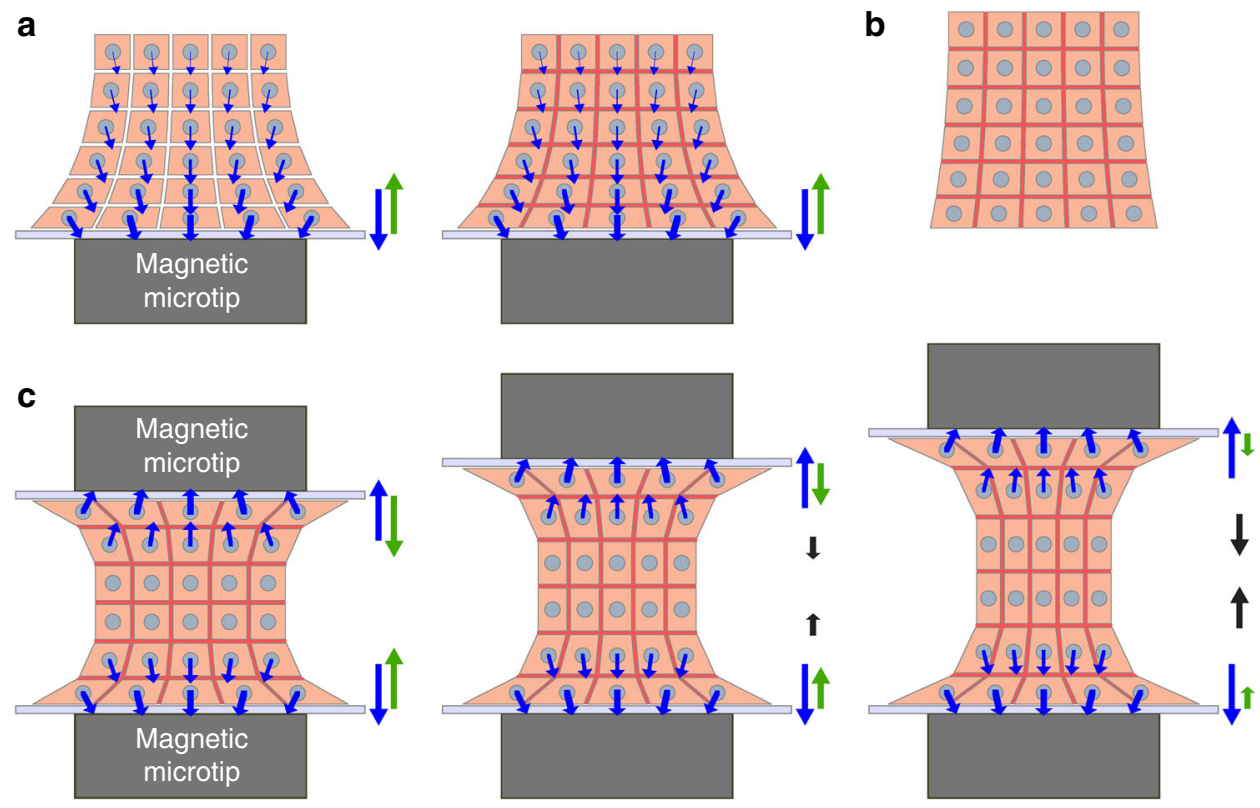

Fig. 7 Schematic view of the forces involved within the EB in the magnetic stretcher. a Formation of the EB on the magnetic microtip located below a glass wall. Each cell is subjected to a magnetic force (blue arrow). The total resulting magnetic force (shown on the right-hand side, also in blue) is exactly balanced by the wall reaction force (green arrow). This pair of forces act like a "clamp" that holds mainly the "proximal" region of the sample, closest to the glass wall. After the magnetic contact, adhesion molecules (in red) develop the EB cohesion, without affecting forces. b The whole aggregate can then be used as a standalone EB. c When another magnetic microtip is approached with another glass wall, the upper cell layers are "clamped" against the upper wall in a similar way as in a. Varying the separation of both "clamps" makes it possible to adjust or cycle the (tensile) strain of the main part of the EB (represented here with a thickness of only two cells for simplicity, but actually corresponding to the major part of the entire EB)

while using two magnets, whether in the stretching (static) or cyclic condition, clearly upregulate some genes (Fig. 6a, c). With just one magnetic microtip (Fig. 7a), the tip behaves as a clamp holding a limited (proximal) region of the $\mathrm{EB}$, the only region where substantial forces are present. Indeed, the applied magnetic forces and resulting compression strongly decay with distance from the tip. The major part of the EB thus undergoes negligible stress in this one-magnet situation, and overall gene expression is not affected. By contrast, in the two-magnet situation, the major part of the EB is stretched (Fig. 7c). As a result, gene expression is expected to be altered in most cells, as detected with global PCR measurement.

The magnetic stretcher brings several unique advantages: a 3D aggregate composed solely of its component cells can be formed in situ, without a supporting matrix, and can then be deformed and stimulated, with no transfer step, simply by applying remote magnetic forces. This remote deformation is perfectly illustrated by the immediate release of the tissue when the magnets are removed, and represents another advantage of the approach: the stimulated tissue is easily recovered, perfectly intact. Moreover, it is important to note that this approach is theoretically feasible with any cell type capable of cell-cell adhesion to form a cohesive assembly.

Finally, we show that remote magnetic stimulation (stretching and cyclic stretching) can promote cardiac differentiation without the need of chemical factors. In brief, we found that the stimulation significantly increased the expression of the Gata4, Gata6, Sox17 and Nkx2.5 genes, and that cyclic stimulation had an even more important effect, at day 5 of differentiation. The pattern of these increases is consistent with cardiac mesoderm differentiation (Supplementary Fig. 8). First, the cardiac mesoderm marker gene $N k \times 2.5$ is importantly upregulated. Then, Gata4 and Gata6, endo/mesoderm genes are also upregulated, and were described to play a decisive role in cardiac differentiation ${ }^{59}, 60$. Finally Sox 17 is overexpressed here as described during cardiomyogenesis ${ }^{61}$. Besides, we then demonstrated that later on, at day 10 of differentiation, later cardiac markers such as troponin T (Tnnt2), cardiac $\alpha$-actin (Actc1), $\alpha$ myosin heavy chain (Myh6), and myosin regulatory light chain $2(M y l 2)$ were upregulated as well by the stimulation. The use of the magnetic stretcher therefore revealed that mechanical deformation is by itself sufficient to enhance ESC differentiation towards a cardiac phenotype. 2D cell cyclic stretching was similarly found to improve cardiac differentiation and maturation of cardiomyocytes ${ }^{16,62}$.

Here we do not provide any molecular mechanisms for the cardiac commitment. However, because the mechanical stimulation is that of a global strain applied to the EB, and resembles the situation of a mechanical stretching on a $2 \mathrm{D}$ deformable substrate, we can extrapolate from these works how stem cells may translate external forces to mesodermal differentiation. In brief, mechanical stimuli such as stretch and shear stress can activate several signaling pathways such as PI3K/Akt, ERK1/2, GSK-3ß, Tgf- $\beta^{63}$, Fgf5 ${ }^{64}$, and in turn initiate a cardiovascular differentiation program ${ }^{65-67}$, or facilitate cell-cell connections $^{68}$.

To conclude, we proposed here a technology capable of creating embryoid bodies (EBs) from individual embryonic stem cells (ESCs) through the use of remote magnets, while maintaining the cells' functionality and differentiation profile. This approach is an interesting alternative to conventional techniques for EBs' formation, such as the hanging drop method. The magnetic stretching and stimulation of the resulting EBs then provided a tool to explore the impact of physical biosensing on ESCs differentiation. This purely mechanical stretching enhanced the EBs towards the cardiac mesoderm differentiation pathway, and this was even more pronounced with the application of cyclic stimulation mimicking heart muscle contraction. 


\section{Methods}

ESC culture. CGR8 cell line was purchased from Sigma-Aldrich (09C028). This cell line was established from the inner cell mass of a 3.5 day male pre-implantation mouse embryo (Mus musculus, strain 129). Regular monitoring of cell cultures for Mycoplasma infections was performed using VenorGeM OneStep Mycoplasma Detection Kit (Sigma-Aldrich). To maintain their pluripotency, the cells were cultured on $0.1 \%$ gelatin (Sigma-Aldrich)-coated tissue culture plates in Glasgow's modified Eagle's medium (GMEM, Thermo Fisher Scientific) supplemented with $10 \%$ fetal calf serum (Thermo Fisher Scientific), $2 \mathrm{mM}$ glutamine (Thermo Fisher Scientific), $1 \%$ nonessential amino acids (Thermo Fisher Scientific), $0.1 \mathrm{mM}$ $\beta$-mercaptoethanol (Sigma-Aldrich), $1 \mathrm{mM}$ sodium pyruvate (Thermo Fisher Scientific), $1 \%$ penicillin/streptomycin (Thermo Fisher Scientific); $1000 \mathrm{U} / \mathrm{ml}$ of leukemia inhibitor factor (LIF, Thermo Fisher Scientific) were added prior to use. The cells were cultured at the density of $1.2-10^{4} / \mathrm{cm}^{2}$ at $37^{\circ} \mathrm{C}$ in a humidified $5 \%$ CO2-95\% air atmosphere and passaged every 2 days.

Iron oxide magnetic nanoparticles. Iron oxide nanoparticles (provided by PHENIX, UMR 8234, Paris) were synthetized by alkaline coprecipitation of $\mathrm{FeCl} 2$ $(0.9 \mathrm{~mol})$ and $\mathrm{FeCl} 3(1.5 \mathrm{~mol})$ salts. The nanoparticles were then oxidized into maghemite with $1.3 \mathrm{~mol}$ of iron nitrate under boiling. After magnetic decantation, the maghemite nanoparticles were heated at $80^{\circ} \mathrm{C}$ for $30 \mathrm{~min}$ in water, then supplemented with sodium citrate $(70 \mathrm{~g})$ to promote absorption of citrate anions onto their surface (to ensure electrostatic stabilization in aqueous solution) before precipitation in acetone at $25^{\circ} \mathrm{C}$ and resuspension in water. The resulting nanoparticles were $8 \mathrm{~nm}$ in diameter, with polydispersity index of $35 \%$.

Magnetic cell labeling. Before their incubation with ESCs, the nanoparticles were dispersed at final $[\mathrm{Fe}]$ concentrations between 0.2 and $5 \mathrm{mM}$ in RPMI (Thermo Fisher Scientific) supplemented with citrate at a final concentration of $5 \mathrm{mM}$ to prevent nanoparticle aggregation. The ESCs were incubated in this medium between $30 \mathrm{~min}$ and $4 \mathrm{~h}$, rinsed twice with RPMI medium, and then returned to complete proliferation medium (containing LIF) for at least $2 \mathrm{~h}$ before use.

\section{Determination of the cell iron mass by magnetophoresis. The mass of iron} incorporated by the cells was determined by magnetophoresis, based on the determination of radius $\left(\mathrm{R}_{\text {cell }}\right)$ and velocity $\left(\mathrm{v}_{\text {cell }}\right)$ of single cells dispersed in aqueous medium (viscosity $\eta=10^{3}$ Pa.s) and attracted by a magnet whose field and gradient were perfectly calibrated $(B=0.15 \mathrm{~T} ; \operatorname{gradB}=17 \mathrm{~T} / \mathrm{m})$. Calculation of the cellular iron mass is straightforward: In the horizontal plane of cell movement, the magnetic force $\left(\mathrm{M}_{\text {cell }}\right.$ gradB $)$ is counterbalanced by Stokes' viscous force $\left(6 \pi \eta \mathrm{R}_{\text {cell }} \mathrm{V}_{\text {cell }}\right)$, thus providing the magnetic moment $\left(\mathrm{M}_{\text {cell }}\right)$ of the analyzed cell, which can be transformed into the mass of iron internalized via the volumic magnetization $(50 \mathrm{emu} / \mathrm{g}$ for the field $\mathrm{B}$ of $0.15 \mathrm{~T})$. For each magnetophoretic measurement, the radius and velocity of 200 cells were measured using image processing (Image J). The cellular iron mass (in pg of iron per cell) obtained corresponds to the average value for the cell population (distribution of about $35 \%)$, itself averaged over at least 3 independent experiments.

Perls staining of intracellular iron. Perls' Prussian blue staining was used to reveal iron-labeled cells in blue. The cells were fixed in $10 \%$ formalin solution in PBS then rinsed with PBS and incubated for $30 \mathrm{~min}$ at room temperature with $1 \%$ potassium ferrocyanide in $1 \%$ aqueous solution of hydrochloric acid. They were then rinsed with PBS and observed by optical transmission microscopy.

Cell viability testing. The Alamar Blue metabolic test (Thermo Fisher Scientific) was used to assess the impact of the magnetic nanoparticles on ESC viability. The active ingredient, resazurin, becomes highly fluorescent when metabolized. Fluorescence was quantified with a spectrophotometer (excitation $530 \mathrm{~nm}$, emission $590 \mathrm{~nm}$ ), and the values were interpreted relative to control values (unlabeled cells in complete medium) obtained under similar conditions.

\section{EB magnetophoresis for monitoring nanoparticles fate. To measure the} magnetic moment $(\mathrm{M})$ of the EBs, single EBs were immersed at each different time point after formation (days $1,2,4$, and $7, n>8$ for each condition) in a glycerol solution ( $80 \%$, room temperature $23-24^{\circ} \mathrm{C}$, viscosity $\eta=0.05$ Pa.s) submitted to a magnetic field gradient $(B=150 \mathrm{mT}$, gradB $=17.5 \mathrm{~T} / \mathrm{m})$ generated by a permanent magnet (cylinder $25 \mathrm{~mm}$ in diameter, $10 \mathrm{~mm}$ height). Each EB thus experiences a magnetic velocity $\mathrm{v}_{\text {mag }}$ towards the magnet, by balancing the magnetic force MgradB, and the Stokes drag force $6 \pi \eta \mathrm{Rv}_{\text {mag }}$, where $\mathrm{R}$ is the $\mathrm{EB}$ radius. $\mathrm{EB}$ migration was video-monitored every $0.1 \mathrm{~s}(\times 4$ objective, Leica DMIRB microscope). The magnetic moment calculated (in A. $\mathrm{m}^{2}$, at $150 \mathrm{mT}$ ) can be converted to grams of (magnetic) iron $\left(68 \mathrm{emu} / \mathrm{g}\right.$ at $\left.150 \mathrm{mT}, 1 \mathrm{~A} \cdot \mathrm{m}^{2}=10^{3} \mathrm{emu}\right)$.

Relative quantification of gene expression by real-time PCR. Total RNA was isolated using NucleoSpin RNA kit (Machery-Nagel) according to the manufacturer's instructions. To avoid genomic DNA contamination, RNA samples were incubated for $15 \mathrm{~min}$ with $10 \mathrm{U}$ of DNase. Complementary DNA (cDNA) was then synthesized using SuperScript II Reverse Transcriptase kit (Thermo Fisher
Scientific) with random hexamers according to the manufacturer's instructions. Real-time PCR analysis was then carried out with SYBR green PCR technology using the StepOnePlus system (Thermo Fisher Scientific). The expression of $60 \mathrm{~S}$ acidic ribosomal protein P0 (RPLP0) was used as a reference transcript. All sequences of primers used are presented in Supplementary Table 1.

EB formation by the hanging drop method. Two days after passage, cells were detached, centrifuged and resuspended in differentiation medium (GMEM supplemented with $20 \%$ fetal calf serum, $2 \mathrm{mM}$ glutamine, $1 \%$ nonessential amino acids, $0.1 \mathrm{mM} \beta$-mercaptoethanol, $1 \mathrm{mM}$ sodium pyruvate, $1 \%$ penicillin/streptomycin). Note that the LIF factor must be removed from culture medium since day 0 of EBs formation, resulting in initiation of differentiation. Drops containing 1000 or 10,000 cells in $30 \mu \mathrm{l}$ of medium were plated as hanging drops on a lid of $10 \mathrm{~cm}$ non-adherent Petri dish, which was then inverted over the bottom of the dish filled with PBS to prevent drying. After 2 days (D0-D2), the aggregates thus formed were transferred to a Petri dish containing $10 \mathrm{ml}$ of differentiation medium for a 3-day maturation period (D2-D5). Mature aggregates were finally transferred to $0.1 \%$ gelatin-coated 24-well dishes. The medium was changed every 2-3 days.

EB formation by magnetic attraction. Cells labeled with magnetic nanoparticles were detached, centrifuged and resuspended in differentiation medium. They were then placed in a glass-bottom Petri dish. To prevent cell adhesion, petri dish was previously incubated for $30 \mathrm{~min}$ with $10 \mathrm{mg} / \mathrm{ml} \mathrm{PLL-PEG} \mathrm{(SuSos)} \mathrm{diluted} \mathrm{in} 10 \mathrm{mM}$ HEPES, then rinsed with sterile water, and placed on a magnetic device composed of several magnetic attractors. The magnetic device fabrication is straightforward. First make holes (typically 9 or 16, arranged in a square 3-4 mm lattice) with $0.8 \mathrm{~mm}$ drill through aluminum cylindrical plates (Dural) $8 \mathrm{~mm}$ thick and $35 \mathrm{~mm}$ diameter to match the size of small Petri dishes; Then take typical sewing pin, to be used as soft-iron cylinders with a diameter of $750 \mu \mathrm{m}$. Insert the pins in the holes, and cut at the plate surface (use a drilling machine to level the surface; Place this magnetic pins array over a permanent magnet (typically disc neodymium magnet $\varnothing 20 \mathrm{~mm}$ diameter, $8 \mathrm{~mm}$ height, strength about $10 \mathrm{~kg}$, magnetic field created at the surface $\sim 0.4 \mathrm{~T}$ ). The device is ready to be used. Place it over a Petri dish with glass bottom, and deposit the ESCs in culture medium. The number of deposited cells was adjusted to obtain between 1000 and 30,000 cells per magnetic microtip. The magnetic device was subsequently removed between $5 \mathrm{~min}$ and 2 days, depending on the experiment.

Formation and stimulation of EBs in the magnetic stretcher. The magnetic stretcher is described in detail in the results section. It consists of a reservoir with two magnetic structures, one fixed and the other mobile, each comprising 3 magnetic microtips and the 3 magnets used to magnetize them. The principle is to form 3 EBs over the 3 fixed magnetic microtips of the fixed structure, and then stretch them by the attractive force of the 3 matching microtips on the mobile structure. They could also be stimulated at will by subjecting the second structure to micro-controlled movements.

The reservoir was first washed in $70 \%$ ethanol for $15 \mathrm{~min}$, then dried and rinsed 3 times with sterile water before being exposed to UV for $30 \mathrm{~min}$. The glass slides protecting the magnetized microtips were coated with PLL-PEG to prevent cell adhesion. Before use, the stretching device was sterilized by UV and placed in a sterile box, with a sterile cover placed over the reservoir.

Three EBs, each composed of 10,000 ESCs (labeled with $2 \mathrm{mM}$ iron for $30 \mathrm{~min}$ ), were formed on day 0 on the fixed set of magnetic microtips. One hour later they were stretched, at amplitude corresponding to $50 \%$ of their original size, by approaching the mobile set of magnetic tips (stretched condition). The whole assembly was then placed overnight in an incubator $\left(37^{\circ} \mathrm{C}, 5 \% \mathrm{CO}_{2}\right)$. In some cases (cyclic condition), starting the following day (day 1), the EBs were stimulated twice daily for $2 \mathrm{~h}$ at a frequency of $1 \mathrm{~Hz}$ and an amplitude corresponding to $10 \%$ of the initial height of the aggregate. Stimulation was applied for the next 3 days (day 1 -day 3 ). At the end of day 3 , the microtips were demagnetized by removing the permanent magnets. This instantly released EBs were transferred to Petri dishes and again allowed to mature for 2 days (up to day 5 ) before analysis.

Fluorescence live imaging. Cell membranes were stained with a red fluorochrome Pkh26 from Sigma. Cell stimulation with a $10 \%$ strain applied at $1 \mathrm{~Hz}$ was observed in situ, on living cells, by fluorescence microscopy.

Velocity mapping: The PIV analysis was computed using the Matpiv software package (a GNU public license software) for MATLAB (The MathWorks, Natick, $\mathrm{MA})^{69}, 70$. We used $64 \times 64$-pixels $(40 \times 40 \mu \mathrm{m})$ interrogation windows with $75 \%$ overlap. Calculation of the correlation between two successive subwindows was performed by fast Fourier transform (the single method). Aberrant vectors were filtered out from the velocity fields with a median Gaussian filter.

Immunohistology. 16- $\mu$ m cryosections of EBs were fixed with $4 \%$ PFA (Interchim) during $5 \mathrm{~min}$, washed with PBS, and then incubated 5 min with PBS-Triton X-100 $(0.1 \% \mathrm{v} / \mathrm{v})$. Nonspecific sites were blocked with $5 \%(\mathrm{w} / \mathrm{v})$ bovine serum albumin (BSA) diluted in PBS during 1 hour and incubated with primary antibodies overnight at $4{ }^{\circ} \mathrm{C}$. Anti-Brachyury T (Abcam, 1:200) and anti-Nkx2.5 (Santa Cruz Technologies, 1:100) primary antibodies were used. The binding of primary 
antibodies was detected by incubation for $3 \mathrm{~h}$ with Alexa Fluor-conjugated antirabbit IgG (Cell Signaling, 1:1000). Finally, cells were washed in PBS and mounted with Prolong ${ }^{\circledR}$ Diamond Antifade Mounting Medium with DAPI (Life Technology) for nuclear staining. Cells were analyzed with an Olympus JX81/BX61 device/ Yokogawa CSU device spinning-disk microscope (Andor Technology), equipped with a $63 \mathrm{X}$ oil objective (Olympus).

Statistical analysis. All measurements were made at least three times, in independent conditions. All results are shown as the mean + standard error of the mean (SEM). Parametric student's $t$ test, two-sided, was used to compare the mean of two values obtained for two independent conditions; ${ }^{*} p<0.05$ indicates a significant result, ${ }^{* *} p<0.01$ a very significant result, and ${ }^{* *} p<0.001$ a highly significant result.

Data availability. Data supporting the findings of this study are available within the article and its Supplementary information files, and from the corresponding author upon reasonable request.

Received: 9 November 2016 Accepted: 6 July 2017

Published online: 12 September 2017

\section{References}

1. Pati, F. et al. Printing three-dimensional tissue analogues with decellularized extracellular matrix bioink. Nat. Commun. 5, 3935 (2014).

2. Giobbe, G. G. et al. Functional differentiation of human pluripotent stem cells on a chip. Nat. Meth. 12, 637-640 (2015).

3. Todhunter, M. E. et al. Programmed synthesis of three-dimensional tissues. Nat. Methods 12, 975-981 (2015).

4. Parmar, P. A. et al. Temporally degradable collagen-mimetic hydrogels tuned to chondrogenesis of human mesenchymal stem cells. Biomaterials $99,56-71$ (2016).

5. Discher, D. E., Mooney, D. J. \& Zandstra, P. W. Growth factors, matrices, and forces combine and control stem cells. Science 324, 1673-1677 (2009).

6. Engler, A. J., Sen, S., Sweeney, H. L. \& Discher, D. E. Matrix elasticity directs stem cell lineage specification. Cell 126, 677-689 (2006).

7. Evans, N. D. et al. Substrate stiffness affects early differentiation events in embryonic stem cells. Eur. Cell. Mater. 18, el3 (2009).

8. Gobaa, S., Hoehnel, S. \& Lutolf, M. Substrate elasticity modulates the responsiveness of mesenchymal stem cells to commitment cues. Int. Biol. 7 , 1135-1142 (2015).

9. Sun, Y. et al. Hippo/YAP-mediated rigidity-dependent motor neuron differentiation of human pluripotent stem cells. Nat. Mater 13, 599-604 (2014).

10. Keung, A. J., Asuri, P., Kumar, S. \& Schaffer, D. V. Soft microenvironments promote the early neurogenic differentiation but not self-renewal of human pluripotent stem cells. Int. Biol. 4, 1049-1058 (2012).

11. Przybyla, L., Lakins, J. N. \& Weaver, V. M. Tissue Mechanics Orchestrate Wnt-Dependent Human Embryonic Stem Cell Differentiation. Cell Stem Cell 19, 462-475 (2016)

12. Huang, Y. et al. Effect of cyclic strain on cardiomyogenic differentiation of rat bone marrow derived mesenchymal stem cells. PLoS ONE 7, e34960 (2012).

13. Adamo, L. et al. Biomechanical forces promote embryonic haematopoiesis. Nature. 459, 1131-1135 (2009).

14. Yamamoto, K. et al. Fluid shear stress induces differentiation of Flk-1-positive embryonic stem cells into vascular endothelial cells in vitro. Am. J. Physiol. 288, H1915-H1924 (2005).

15. Geuss, L. R. \& Suggs, L. J. Making cardiomyocytes: how mechanical stimulation can influence differentiation of pluripotent stem cells. Biotechnol. Prog. 29, 1089-1096 (2013).

16. Gwak, S.-J. et al. The effect of cyclic strain on embryonic stem cell-derived cardiomyocytes. Biomaterials 29, 844-856 (2008).

17. Kurpinski, K., Chu, J., Hashi, C. \& Li, S. Anisotropic mechanosensing by mesenchymal stem cells. Proc. Natl Acad. Sci. USA 103, 16095-16100 (2006).

18. Chowdhury, F. et al. Material properties of the cell dictate stress-induced spreading and differentiation in embryonic stem cells. Nat. Mater 9, 82-88 (2010).

19. Uda, Y. et al. Force via integrins but not E-cadherin decreases Oct3/4 expression in embryonic stem cells. Biochem. Biophys. Res. Commun. 415, 396-400 (2011)

20. Pelaez, D., Charles Huang, C.-Y. \& Cheung, H. S. Cyclic compression maintains viability and induces chondrogenesis of human mesenchymal stem cells in fibrin gel scaffolds. Stem Cells Dev. 18, 93-102 (2009).

21. Henstock, J. \& El Haj, A. Controlled mechanotransduction in therapeutic MSCs: can remotely controlled magnetic nanoparticles regenerate bones? Regen. Med. 10, 377-380 (2015).
22. Matthys, O. B., Hookway, T. A. \& McDevitt, T. C. Design Principles for Engineering of Tissues from Human Pluripotent Stem Cells. Curr. Stem Cell Rep. 2, 43-51 (2016).

23. Poh, Y.-C. et al. Generation of organized germ layers from a single mouse embryonic stem cell. Nat. Commun. 5, 4000 (2014).

24. Bratt-Leal, A. M., Kepple, K. L., Carpenedo, R. L., Cooke, M. T. \& McDevitt, T. C. Magnetic manipulation and spatial patterning of multi-cellular stem cell aggregates. Int. Biol. 3, 1224-1232 (2011).

25. Mironov, V. et al. Organ printing: tissue spheroids as building blocks. Biomaterials 30, 2164-2174 (2009).

26. Ahrens, E. T. \& Bulte, J. W. Tracking immune cells in vivo using magnetic resonance imaging. Nat. Rev. Immunol. 13, 755-763 (2013).

27. Cromer Berman, S. M., Walczak, P. \& Bulte, J. W. Tracking stem cells using magnetic nanoparticles. Wiley Interdiscip. Rev. Nanomed. Nanobiotechnol. $\mathbf{3}$, 343-355 (2011).

28. Harrison R. et al. Autonomous magnetic labelling of functional mesenchymal stem cells for improved traceability and spatial control in cell therapy applications. J. Tissue Eng. Regen. Med. doi:10.1002/term.2133 (2016)

29. Tang, Y. et al. MRI/SPECT/fluorescent tri-modal probe for evaluating the homing and therapeutic efficacy of transplanted mesenchymal stem cells in a rat ischemic stroke model. Adv. Funct. Mater 25, 1024-1034 (2015).

30. El Haj, A. J. et al. An in vitro model of mesenchymal stem cell targeting using magnetic particle labelling. J. Tissue Eng. Regen. Med. 9, 724-733 (2015).

31. Landázuri, N. et al. Magnetic targeting of human mesenchymal stem cells with internalized superparamagnetic iron oxide nanoparticles. Small 9, 4017-4026 (2013).

32. Cheng, K. et al. Magnetic enhancement of cell retention, engraftment, and functional benefit after intracoronary delivery of cardiac-derived stem cells in a rat model of ischemia/reperfusion. Cell Transplant 21, 1121-1135 (2012).

33. Kim, J. A. et al. High-throughput generation of spheroids using magnetic nanoparticles for three-dimensional cell culture. Biomaterials 34, 8555-8563 (2013).

34. Mattix, B. et al. Biological magnetic cellular spheroids as building blocks for tissue engineering. Acta Biomater. 10, 623-629 (2014).

35. Souza, G. R. et al. Three-dimensional tissue culture based on magnetic cell levitation. Nat. Nanotech. 5, 291-296 (2010).

36. Lee J.-K., Ito A., Honda H. Construction of functional cardiovascular tissues using magnetic nanoparticles. Cardiac Regeneration Using Stem Cells, 221-228 (2013).

37. Kito, T. et al. iPS cell sheets created by a novel magnetite tissue engineering method for reparative angiogenesis. Sci. Rep. 3, 1418 (2013).

38. Arbab, A. S. et al. Labeling of cells with ferumoxides-protamine sulfate complexes does not inhibit function or differentiation capacity of hematopoietic or mesenchymal stem cells. NMR Biomed. 18, 553-559 (2005).

39. Kostura, L., Kraitchman, D. L., Mackay, A. M., Pittenger, M. F. \& Bulte, J. W. Feridex labeling of mesenchymal stem cells inhibits chondrogenesis but not adipogenesis or osteogenesis. NMR Biomed. 17, 513-517 (2004).

40. Fayol, D., Luciani, N., Lartigue, L., Gazeau, F. \& Wilhelm, C. Managing magnetic nanoparticle aggregation and cellular uptake: a precondition for efficient stem-cell differentiation and MRI tracking. Adv. Healthc. Mater 2, 313-325 (2013).

41. Han, J. et al. Iron oxide nanoparticle-mediated development of cellular gap junction crosstalk to improve mesenchymal stem cells' therapeutic efficacy for myocardial infarction. ACS Nano 9, 2805-2819 (2015).

42. Cheng, K. et al. Magnetic antibody-linked nanomatchmakers for therapeutic cell targeting. Nat. Commun. 5, 4880 (2014).

43. Au, K.-W. et al. Effects of iron oxide nanoparticles on cardiac differentiation of embryonic stem cells. Biochem. Biophys. Res. Commun. 379, 898-903 (2009).

44. Parsa, H. et al. Effect of superparamagnetic iron oxide nanoparticles-labeling on mouse embryonic stem cells. Cell J. 17, 221-230 (2016).

45. Mazuel, F. et al. Massive intracellular biodegradation of iron oxide nanoparticles evidenced magnetically at single-endosome and tissue levels. ACS Nano 10, 7627-7638 (2016).

46. Mazuel, F. et al. Magneto-Thermal Metrics Can Mirror the Long-Term Intracellular Fate of Magneto-Plasmonic Nanohybrids and Reveal the Remarkable Shielding Effect of Gold. Adv. Funct. Mat 27, 1605997 (2017).

47. Zhao, R., Boudou, T., Wang, W. G., Chen, C. S. \& Reich, D. H. Decoupling cell and matrix mechanics in engineered microtissues using magnetically actuated microcantilevers. Adv. Mat 25, 1699-1705 (2013).

48. Trepat, X. et al. Physical forces during collective cell migration. Nat. Phys. 5, 426-430 (2009).

49. Frade, A. F. et al. Polymorphism in the alpha cardiac muscle actin 1 gene is associated to susceptibility to chronic inflammatory cardiomyopathy. PLoS ONE 8, e83446 (2013).

50. Chang, Y. K., Liu, Y. P., Ho, J. H., Hsu, S. C. \& Lee, O. K. Amine-surfacemodified superparamagnetic iron oxide nanoparticles interfere with differentiation of human mesenchymal stem cells. J. Orthop. Res. 30, 1499-1506 (2012). 
51. Kurosawa, H. Methods for inducing embryoid body formation: in vitro differentiation system of embryonic stem cells. J. Biosci. Bioeng. 103, 389-398 (2007).

52. Choi, Y. Y. et al. Controlled-size embryoid body formation in concave microwell arrays. Biomaterials. 31, 4296-4303 (2010).

53. Park, J. et al. Microfabrication-based modulation of embryonic stem cell differentiation. Lab. Chip. 7, 1018-1028 (2007).

54. Nakazawa, K., Yoshiura, Y., Koga, H. \& Sakai, Y. Characterization of mouse embryoid bodies cultured on microwell chips with different well sizes. J. Biosci. Bioeng. 116, 628-633 (2013).

55. Ungrin, M. D., Joshi, C., Nica, A., Bauwens, C. \& Zandstra, P. W. Reproducible, ultra high-throughput formation of multicellular organization from single cell suspension-derived human embryonic stem cell aggregates. PLOS ONE 3, e1565 (2008).

56. Van Winkle, A. P., Gates, I. D. \& Kallos, M. S. Mass transfer limitations in embryoid bodies during human embryonic stem cell differentiation. Cells Tissues Organs 196, 34-47 (2012).

57. Blancas, A. A., Chen, C.-S., Stolberg, S. \& McCloskey, K. E. Adhesive forces in embryonic stem cell cultures. Cell Adhes. Migr. 5, 472-479 (2011).

58. Harris, A. R. et al. Characterizing the mechanics of cultured cell monolayers Proc. Natl Acad. Sci. USA 109, 16449-16454 (2012).

59. Xin, M. et al. A threshold of GATA4 and GATA6 expression is required for cardiovascular development. Proc. Natl Acad. Sci. USA 103, 11189-11194 (2006).

60. Zhao, R. et al. Loss of both GATA4 and GATA6 blocks cardiac myocyte differentiation and results in acardia in mice. Dev. Biol. 317, 614-619 (2008).

61. Stefanovic, S. et al. Interplay of Oct4 with Sox 2 and Sox17: a molecular switch from stem cell pluripotency to specifying a cardiac fate. J. Cell Biol. 186, 665-673 (2009).

62. Zhang, T. et al. Channelled scaffolds for engineering myocardium with mechanical stimulation. J. Tissue Eng. Regen. Med. 6, 748-756 (2012).

63. Banerjee, I. et al. Cyclic stretch of embryonic cardiomyocytes increases proliferation, growth, and expression while repressing Tgf- $\beta$ signaling. J. Mol. Cell Cardiol. 79, 133-144 (2015).

64. Toh, Y.-C. \& Voldman, J. Fluid shear stress primes mouse embryonic stem cells for differentiation in a self-renewing environment via heparan sulfate proteoglycans transduction. FASEB J. 25, 1208-1217 (2011).

65. Pan, J. et al. Mechanical stretch activates the JAK/STAT pathway in rat cardiomyocytes. Circ. Res. 84, 1127-1136 (1999).

66. Kim, C.-H., Cho, Y.-S., Chun, Y.-S., Park, J.-W. \& Kim, M.-S. Early expression of myocardial HIF- $1 \alpha$ in response to mechanical stresses. Circ. Res. 90, e25-e33 (2002).

67. Seko, Y. et al. Pulsatile stretch activates mitogen-activated protein kinase (MAPK) family members and focal adhesion kinase (p125FAK) in cultured rat cardiac myocytes. Biochem. Biophys. Res. Commun. 259, 8-14 (1999).

68. Salameh, A. et al. Cyclic mechanical stretch induces cardiomyocyte orientation and polarization of the gap junction protein connexin43. Circ. Res. 106, 1592-1602 (2010).
69. Petitjean, L. et al. Velocity fields in a collectively migrating epithelium. Biophys. J. 98, 1790-1800 (2010).

70. Vedula, S. R. K. et al. Epithelial bridges maintain tissue integrity during collective cell migration. Nat. Mater 13, 87-96 (2014).

\section{Acknowledgements}

This work was supported by the European Union (ERC-2014-CoG project MaTissE 648779), and French National Research Agency (MagStem ANR-11 SVSE5 \& LabEx REVIVE ANR-10-LABX-73). We thank Alexandre Lantheaume for mechanical assistance, Aude Michel for providing us with the nanoparticles (PHENIX, UMR 8234, Paris), and Christine Péchoux for TEM imaging (platform TEM, INRA, Jouy-en-Josas), France).

\section{Author contributions}

N.L., P.M., O.A. and C.W. conceived the idea; V.D., N.L., S.R., G.M., F.M., C.G., M.R. and C.W. performed the experiments; N.L., M.R., O.A., and C.W. supervised the study; C.W. wrote the manuscript; V.D., N.L., S.R., M.R., O.A., C.G., and P.M. proofread the manuscript.

\section{Additional information}

Supplementary Information accompanies this paper at doi:10.1038/s41467-017-00543-2.

Competing interests: The authors declare no competing financial interests.

Reprints and permission information is available online at http://npg.nature.com/ reprintsandpermissions/

Publisher's note: Springer Nature remains neutral with regard to jurisdictional claims in published maps and institutional affiliations.

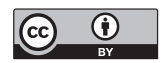

Open Access This article is licensed under a Creative Commons Attribution 4.0 International License, which permits use, sharing, adaptation, distribution and reproduction in any medium or format, as long as you give appropriate credit to the original author(s) and the source, provide a link to the Creative Commons license, and indicate if changes were made. The images or other third party material in this article are included in the article's Creative Commons license, unless indicated otherwise in a credit line to the material. If material is not included in the article's Creative Commons license and your intended use is not permitted by statutory regulation or exceeds the permitted use, you will need to obtain permission directly from the copyright holder. To view a copy of this license, visit http://creativecommons.org/ licenses/by/4.0/.

(c) The Author(s) 2017 